\title{
Efecto del termofosfato sobre el crecimiento y producción de papa (Solanum tuberosum L.)
}

\section{Effect of thermophosphate on the growth and production of potatoes (Solanum tuberosum L.)}

\begin{abstract}
David Fernando Torres-Hernandez ${ }^{1}$; Elberth Hernando Pinzón-Sandoval ${ }^{2}$; Fernando Javier Peña-Baracaldo ${ }^{3}$;
\end{abstract} Saúl Fernando Torres-Rodríguez ${ }^{4}$; Diego Jimenez-Diaz ${ }^{5}$

1'Ing. Agrónomo, M.Sc., Estudiante de Doctorado. Universidad de los Llanos. Villavicencio - Meta, Colombia; e-mail: mollisol89@hotmail.com; Dhttps:// orcid.org/0000-0002-2167-1175

${ }^{2}$ Ing. Agrónomo, M.Sc. Universidad Pedagógica y Tecnológica de Colombia, Grupo de Investigación en Desarrollo y Producción Agraria Sostenible GIPSO. Tunja - Boyacá, Colombia; e-mail: elberth02@gmail.com; Dhttps://orcid.org/0000-0001-9229-3450

${ }^{3}$ Ing. Agrónomo, M.Sc. Universidad de Ciencias Aplicadas y Ambientales U.D.C.A; e-mail: fepena@udca.edu.co; Dhttps://orcid.org/0000-0001-7809-9686

${ }^{4}$ Ing. Agrónomo, Estudiante de Maestría. Universidad Pedagógica y Tecnológica de Colombia. Tunja - Boyacá, Colombia; email: saultorresagro64@gmail. com; (Dhttps://orcid.org/0000-0002-6497-976X

${ }^{5}$ Ing. Agrónomo, Estudiante de Maestría. Universidad Pedagógica y Tecnológica de Colombia. Tunja - Boyacá, Colombia; e-mail: diego.jimenez.diaz@gmail. com; (Dhttps://orcid.org/0000-0002-3358-6832

Cómo citar: Torres-Hernandez, D.F.; Pinzón-Sandoval, E.H.; Peña-Baracaldo, F.J.; Torres-Rodríguez, S.F.; Jimenez-Diaz, D. 2020. Efecto del termofosfato sobre el crecimiento y producción de papa (Solanum tuberosum L.). Rev. U.D.C.A Act. \& Div. Cient. 23(2):e1724. http://doi.org/10.31910/rudca.v23.n2.2020.1724

Artículo de acceso abierto publicado por Revista U.D.C.A Actualidad \& Divulgación Científica, bajo una licencia Creative Commons CC BY-NC 4.0

Publicación oficial de la Universidad de Ciencias Aplicadas y Ambientales U.D.C.A, Institución de Educación Superior Acreditada de Alta Calidad por el Ministerio de Educación Nacional.

Recibido: Septiembre 17 de 2019 Aceptado: Septiembre 29 de 2020 Editado por: Ingeborg Zenner de Polanía

\section{RESUMEN}

El cultivo de papa es uno de los más representativos de los Andes colombianos. El cultivo tiene altos costos de producción, entre los cuales, la fertilización representa un $36 \%$ del total. Los avances tecnológicos en nutrición vegetal han permitido el desarrollo de nuevos compuestos, que generan una mayor asimilación y disponibilidad de los elementos nutritivos, durante los periodos productivos. Dentro de las diferentes fuentes de fósforo empleadas en Colombia, está como alternativa, el termofosfato o fosfato térmico. Por esto, el objetivo del presente trabajo fue evaluar la aplicación del termofosfato en el cultivo de papa variedad
'Superior', en condiciones de campo, en el municipio de Villa Pinzón, Cundinamarca. Se empleó un diseño completamente al azar, con 4 tratamientos, que correspondieron a diferentes porcentajes de fosfato térmico 100\% fosfato diamónico (DAP) (manejo convencional); $80 \%$ DAP, 20\% fosfato térmico (FT); 40\% DAP, 60\% FT y $20 \%$ DAP, $80 \%$ FT, $\left(0,300,600\right.$ y $\left.900 \mathrm{~kg} \mathrm{ha}^{-1}\right)$, con tres réplicas, para un total de 12 unidades experimentales. Las variables evaluadas fueron pesos fresco y seco, de parte área y raíz, concentración de fósforo en tejido y rendimiento por calidades y total. Se observó que la aplicación de termofosfato en diferentes concentraciones generó un efecto positivo con diferencias estadísticas $(\mathrm{P} \leq 0,05)$, en todas las variables evaluadas. El tratamiento de $600 \mathrm{~kg} \mathrm{ha}^{-1}$ de termofosfato, se 
muestra como una alternativa para el aporte de fósforo dentro de los planes de fertilización, en busca de una mejor respuesta fisiológica y productiva del cultivo de papa.

Palabras clave: Nutrición mineral; Tubérculo; Macro nutriente; Solanaceae; Variedad superior.

\section{ABSTRACT}

Potato cultivation is one of the most representative activities of the Colombian Andes. The crop has high production costs, among which fertilization represents $36 \%$ of the total Technological advances in plant nutrition have allowed the development of new compounds that generate greater assimilation and availability of nutritional elements during productive periods. Within the different phosphorus sources available in Colombia, thermophosphate exists as an alternative. For this reason, the objective of this research was to evaluate the application of thermophosphate in the potato crop, Superior 'variety, under field conditions in the municipality of Villa Pinzón-Cundinamarca. A completely randomized design was used with 4 treatments that corresponded to different percentages of thermal phosphate: 100\% diammonium phosphate (DAP) (conventional management); $80 \%$ DAP, 20\% thermal phosphate (TF); $40 \%$ DAP, $60 \%$ TF and 20\% DAP, $80 \%$ TF $(0,300,600 \mathrm{y}$ $900 \mathrm{~kg}$ ha-1), with three replicas for a total of 12 experimental units. The variables evaluated were fresh and dry weights of areal and root part, phosphorus concentration in tissue and yield by qualities and the total. It was observed that the application of thermophosphate at different concentrations generated a positive effect, statistically different $(\mathrm{P} \leq 0.05)$ in all the variables evaluated. Thermophosphate, at $600 \mathrm{~kg}$ ha-1 was found to be an alternative for the contribution of phosphorus within the fertilization plans, in search of a better physiological and productive response of the potato crop.

Keywords: Mineral nutrition; Tuber; Macro nutrient; Solanaceae; Variety "Superior".

\section{INTRODUCCIÓN}

En Colombia, la papa (Solanum tuberosum L.), se caracteriza por ser uno de los principales cultivos de ciclo corto. Su producción, se concentra en ocho departamentos, dentro de los que se encuentra, Boyacá. Tiene altos costos de producción, que son difíciles de disminuir sin afectar los rendimientos (Rodríguez \& Rodríguez, 2014); dentro de estos, se encuentran insumos, como semilla, enmiendas, abonos orgánicos, reguladores fisiológicos y fertilizantes edáficos y foliares, de los cuales, la fertilización del cultivo representa un 36\% dentro del total de costos de producción (Fedepapa, 2019). El cultivo de papa es un gran generador de empleo y es fuente de ingresos y sustento alimentario para un gran número de familias campesinas, ubicadas en las zonas de cordillera, entre los $2.000 \mathrm{y}$ $3.000 \mathrm{~m}$ s.n.m. Según pronósticos del Departamento de Estadísticas de Fedepapa, se espera que, para el final de 2020, se logren sembrar más de 132.161 ha de este tubérculo (Fedepapa, 2019). Existen cerca de 30 variedades entre mejoradas y nativas que aún se cultivan, pero solo 10 de ellas cuentan con importancia comercial nacional o regional, dentro de las que se encuentra, Diacol Capiro, Pardas Pastusa Suprema y Superior, Betina e ICA Única (Santos Castellanos et al. 2010).

El costo de la fertilización fosfórica es alto y su eficiencia puede llegar solo a un 20\% (Nústez et al. 2006). Sin duda, existe un interés económico y agronómico por encontrar alternativas más eficaces de suministro de fósforo en el cultivo de papa.

El fósforo promueve el crecimiento de la raíz, la formación rápida de tubérculos y la síntesis de almidón. En suelos fuertemente ácidos, con $\mathrm{pH}$ inferior a 5,5, como el que se presenta en las zonas de producción de papa, el fósforo es fijado y forma precipitados con hierro y aluminio, lo que disminuye su disponibilidad, afectando, de esta forma, procesos, como la translocación de azúcares a los tubérculos y la síntesis de almidón, proceso fundamental en el crecimiento del tubérculo (Gaitán Moreno et al. 2013).

Existe una amplia gama de fertilizantes y de enmiendas fosfóricas, que aportan fósforo a los suelos; uno de ellos es el termofosfato o fosfato térmico, conocido como material fosfórico, sometido a elevadas condiciones de temperatura (Almeyda Ortiz \& Niño Díaz, 2010). Este material contiene concentraciones de calcio y de silicio, provenientes de los materiales que componen la roca fosfórica, de la cual, se obtiene, estimulando la absorción de fósforo, principalmente en suelos de condición ácida (Fernández, 2007). Existe la percepción general que los fertilizantes convencionales son ineficientes en cuanto al aporte de fósforo a los cultivos (Roberts \& Johnston, 2015); sin embargo, se encuentran fuentes no convencionales, como el fosfato térmico, con propiedades de mayor disponibilidad, debido al proceso de rompimiento térmico de la roca fosfórica, lo que genera un material con una solubilidad intermedia, que mejora la disponibilidad a corto y largo plazo y ofrece a la planta un mayor porcentaje de fósforo, en forma asimilable, durante todo su ciclo fenológico (Fernández \& Noguera, 2003). Dichas propiedades han sido evaluadas en cultivos, como Fríjol cv 'TCA Cerinza' (QuintanaBlanco et al. 2017) y Fresa cv 'Albión' (Galindo-López et al. 2018), en los que se observó un mejor desempeño en variables de crecimiento y de producción.

Teniendo en cuenta la escasa información sobre la respuesta de los cultivos a fuentes no convencionales de fósforo, como el termofosfato y resaltando el papel fundamental que tiene este elemento en la calidad y en la producción de los cultivos agrícolas, en este caso la papa, se deben realizar investigaciones conducentes al uso de alternativas de fertilización eficiente.

El objetivo de este estudio fue el de evaluar el efecto de un termofosfato en diferentes dosis y su respuesta sobre el crecimiento y la producción del cultivo de papa (S. tuberosum L.), bajo condiciones de campo, en el municipio de Villa Pinzón, Cundinamarca. 


\section{MATERIALES Y MÉTODOS}

El desarrollo de la investigación, se llevó a cabo en el municipio de Villapinzón, Cundinamarca, en la vereda de Sonsa, sector el Perico, a una altura de $2.900 \mathrm{~m}$ s.n.m., con una temperatura promedio de $13,2^{\circ} \mathrm{C}$ y precipitación promedio anual de $810 \mathrm{~mm}$. La selección del sitio de estudio, se realizó gracias a visitas y muestreos, identificando un suelo con problemas de acidez, altas concentraciones de aluminio intercambiable y baja disponibilidad de bases intercambiables, dentro una zona de alta producción de papa, utilizando como cultivo indicador, papa Parda Pastusa var. 'Superior', uno de los cultivares con mayor área sembrada en el país que, según Nústez et al. (2009), presenta características de adaptación a las condiciones de ensayo y de aceptación en el mercado.
Para evaluar el efecto del fosfato térmico, se realizó un reemplazo parcial de la fuente de fósforo aplicada por el agricultor de forma convencional; para esto, se empleó un diseño completamente al azar (DCA), con cuatro tratamientos, siendo: T1: 100\% fosfato Diamónico (DAP) (manejo convencional); T2: 80\% DAP, 20\% fosfato térmico (FT); T3: 40\% DAP, 60\% FT y T4: 20\% DAP, $80 \%$ FT. Los porcentajes, se ajustaron al requerimiento del cultivo, de acuerdo con lo reportado por García \& Pantoja (1998) y las condiciones del suelo, determinadas mediante análisis fisicoquímico del mismo (Tabla 1); se utilizó una fuente comercial de fosfato térmico, que presenta una composición garantizada de $\mathrm{P}_{2} \mathrm{O}_{5} 20 \%$; $\mathrm{CaO} 28 \% ; \mathrm{MgO} 8,5 \% ; \mathrm{SiO}_{2} 15 \%$.

Tabla 1. Análisis físico-químico de suelo, sitio de investigación.

\begin{tabular}{|c|c|c|}
\hline Propiedades & Cantidad & Unidades \\
\hline Profundidad de muestreo & 20 & $\mathrm{~cm}$ \\
\hline Conductividad Eléctrica & 0,49 & $\mathrm{dS} . \mathrm{m}^{-1}$ \\
\hline $\mathrm{pH}$ & 4,33 & Escala \\
\hline M.O & 11,8 & $\%$ \\
\hline $\mathrm{P}$ & 24,95 & $\mathrm{ppm}$ \\
\hline $\mathrm{Al}$ & 2,9 & $\mathrm{cmol} \mathrm{kg}^{-1}$ de suelo \\
\hline $\mathrm{H}$ & 0,6 & $\mathrm{cmol} \mathrm{kg}^{-1}$ de suelo \\
\hline $\mathrm{Ca}$ & 5.51 & $\mathrm{cmol} \mathrm{kg}^{-1}$ de suelo \\
\hline $\mathrm{Mg}$ & 2,15 & $\mathrm{cmol} \mathrm{kg}^{-1}$ de suelo \\
\hline $\mathrm{K}$ & 1,96 & $\mathrm{cmol} \mathrm{kg}^{-1}$ de suelo \\
\hline $\mathrm{Na}$ & 0,09 & $\mathrm{cmol} \mathrm{kg}^{-1}$ de suelo \\
\hline $\mathrm{Fe}$ & 11,4 & ppm \\
\hline $\mathrm{Mn}$ & 3,46 & ppm \\
\hline $\mathrm{Cu}$ & 0,06 & ppm \\
\hline
\end{tabular}

Cada tratamiento, se repitió tres veces, para un total de 12 unidades experimentales (UE). Se tomaron 3 surcos de $7,5 \mathrm{~m}$ por unidad experimental, con un total de plantas por UE de 45; la distancia de siembra fue de $1 \mathrm{~m}$, entre surcos y $0,5 \mathrm{~m}$, entre plantas.

La aplicación del fosfato térmico, se hizo al momento de la siembra junto con los demás elementos, empleando, como fuente de nitrógeno, Urea; fuente de potasio, $\mathrm{KCl}$ y un producto comercial, que garantizara el suministro de elementos menores. Se aplicó riego por aspersión, según necesidad del cultivo y aplicación de control fitosanitario, con base en monitoreo, pero respetando las prácticas de manejo del agricultor.

Para la medición de las variables, se seleccionaron 5 plantas de los surcos centrales de unidad experimental, con el fin de evitar el efecto de borde. Una vez el cultivo emergió (aproximadamente, a los 20 días), se procedió a tomar las variables de crecimiento y de producción; se evaluó: masa fresca y seca de raíz y parte aérea (tallos y foliolos), mediante una balanza electrónica Acculab VIC 612 de $0,01 \mathrm{~g}$ de precisión y secadas en una estufa Memmert a $70^{\circ} \mathrm{C}$, hasta que alcanzaron peso constante (aproximadamente 96h); contenido foliar de fósforo, a través del método de calcinación, a $600^{\circ} \mathrm{C}$; digestión ácida y valoración, por espectrofotometría visible. Para el rendimiento, se procedió a recolectar los tubérculos, los cuales, se dispusieron en empaques de fibra blanca, con capacidad para 50kg; se clasificaron de forma comercial en dos categorías, primera y segunda, que coincide con la norma técnica colombiana NTC 341, en la que la calidad 1 (primera) corresponde a tubérculos con un diámetro de 60 a 90mm y la calidad 2 (segunda), tubérculos con un diámetro de 45 a $64 \mathrm{~mm}$. Posteriormente, se extrapolaron los datos de peso fresco de tubérculos obtenidos en cada unidad experimental, a tha ${ }^{-1}$, para obtener el rendimiento total.

Los datos obtenidos fueron sometidos a pruebas de normalidad y homogeneidad de varianza, mediante las pruebas de ShapiroWilk y Levene, respectivamente. Comprobados los supuestos, se 
realizó análisis de varianza; las variables que mostraron diferencias estadísticas fueron sometidas a pruebas de comparación de medias de Tukey $(\mathrm{P} \leq 0,05)$. Los análisis se realizaron con el programa estadístico $\mathrm{R}$.

\section{RESULTADOS Y DISCUSIÓN}

Peso radicular: El peso fresco radicular mostró diferencias estadísticas $(\mathrm{P} \leq 0,05)$. Se obtuvo un valor medio de $69,72 \pm 4,47 \mathrm{~g}$, para el tratamiento testigo, mientras que la aplicación del termofosfato, en dosis de 300, 600 y $900 \mathrm{~kg} \mathrm{ha}^{-1}$, presentaron valores medios de $89,81 \pm 4,81,103,23 \pm 1,79$ y $113,5 \pm 8,73 \mathrm{~g}$, respectivamente (Figura 1A). En cuanto al peso seco radicular, se observaron diferencias estadísticas entre tratamientos $(\mathrm{P} \leq 0,05)$. La aplicación de termofosfato generó una respuesta positiva frente a la no aplicación, con valores medios de 21,3, 23,54 y 23,54g, respectivamente, frente al tratamiento testigo, que presentó un valor medio de $17,73 \mathrm{~g}$ (Figura 1B).
El fósforo es parte de moléculas, como el ATP, así como de la formación de membranas celulares, ya que hace parte de los fosfolípidos (IPNI, 1999), que se puede ver reflejado al inicio de la emisión y formación de raíces y, por consiguiente, facilita la absorción de agua y de nutrientes; esto coincide, con lo encontrado en el presente estudio, en el que la aplicación de termofosfato, en diferentes dosis, generó mayor masa fresca y seca de raíces (Figura 1A y B), debido a que este fertilizante, como lo menciona Quintana-Blanco et al. (2017), al ser 50\% hidrosoluble y 50\% citrosoluble, genera una disponibilidad del elemento de una forma más prolongada en el tiempo (lenta liberación), ajustándose a las diferentes etapas fenológicas de la planta.

Según Cuadros \& Gómez (2010), un incremento en la proporción de raíces permite una exploración a capas bajas del suelo; esta respuesta, se traduce en un mejor desempeño en ambientes con baja disponibilidad de nutrientes, por ejemplo, el fósforo; de aquí la importancia de un desarrollo saludable y eficiente.
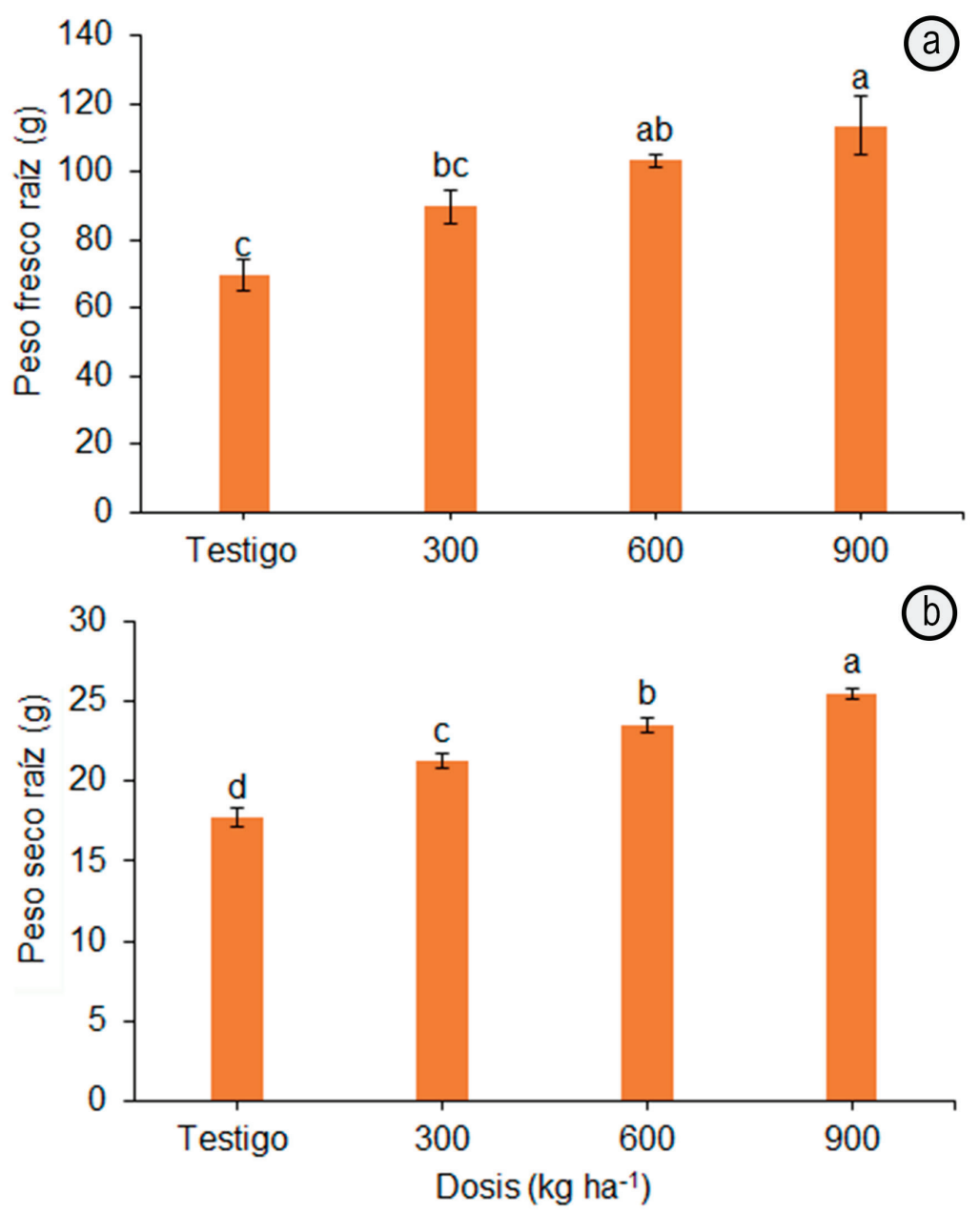

Figura 1. a. Peso fresco radicular; b. Peso seco radicular de plantas de papa (Solanum tuberosum L.) Parda Pastusa variedad 'Superior', con aplicación de termofosfato, en diferentes dosis. Tratamientos seguidos de letras distintas presentan diferencias significativas, según la prueba de Tukey $(\mathrm{P} \leq 0,05)$, barras verticales indican error estándar $(\mathrm{n}=3)$. 
El aumento de masa seca, tanto en la raíz como en la parte aérea, se debe a que el fósforo participa, en gran medida, en la producción de fotoasimilados, según Estrada Ramos (2000); la partición de materia seca es un indicador de la distribución de fotoasimilados hacia los distintos puntos de demanda en crecimiento. Dicho proceso, en el cual está vinculado el fósforo, presenta la salida de las triosas fosfatadas del cloroplasto hacia el citosol, para su posterior transporte como sacarosa, a través del floema (Taiz \& Zeiger, 2010).

Lo observado concuerda con lo reportado por Quintana Blanco et al. (2017), quienes indican que el fósforo juega un papel importante en muchos procesos fisiológicos, como el desarrollo de la plántula y el inicio de la fructificación, representado en aumentos de la materia seca en plantas de fríjol, tras la aplicación de diferentes dosis de P.
Peso aéreo: El peso fresco aéreo presentó diferencias significativas entre tratamientos $(\mathrm{P} \leq 0,05)$. La aplicación del termofosfato mostró un comportamiento diferencial frente a la no aplicación, con valores medios de 966,8 552 a $1657,6 \pm 31 \mathrm{~g}$, respectivamente, en comparación con el tratamiento testigo, el cual, presentó un valor medio de 832,1 $\pm 30 \mathrm{~g}$ (Figura 2A). La aplicación del fosfato térmico mostró un efecto positivo, con diferencias significativas $(\mathrm{P} \leq 0,05)$, entre tratamientos, en el peso seco aéreo. La aplicación de un $600 \mathrm{~kg} \mathrm{ha}^{-1}$ de termofosfato generó un mayor peso seco de la parte

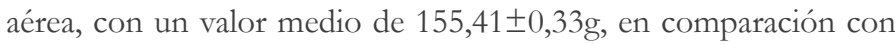
el tratamiento testigo, que mostró un valor medio de $91,03 \pm 0,56 \mathrm{~g}$ (Figura 2B).
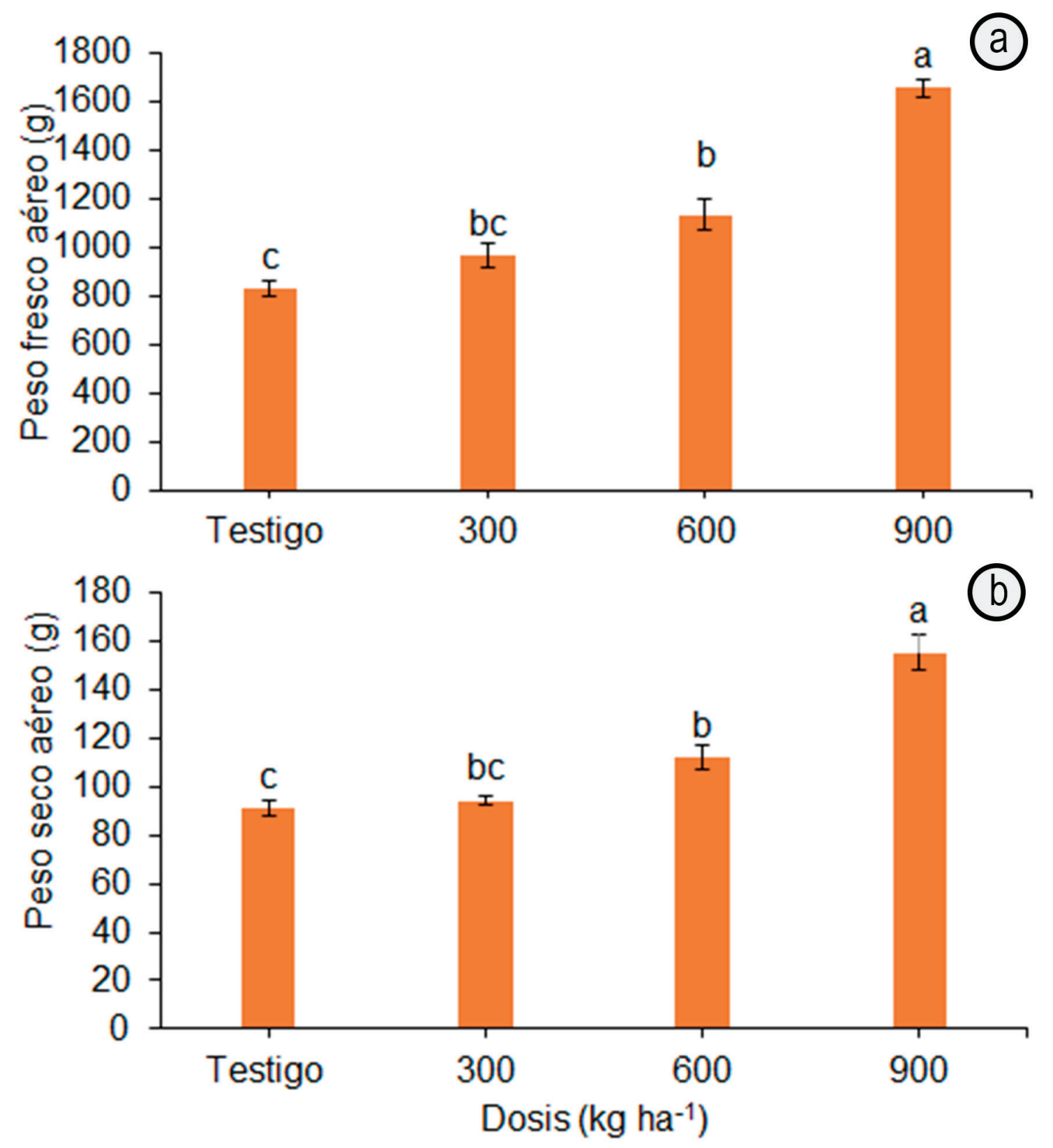

Figura 2. a. Peso fresco aéreo; b. Peso seco aéreo de plantas de papa (Solanum tuberosum L.) Parda Pastusa variedad 'Superior', con aplicación de termofosfato, en diferentes dosis. Tratamientos seguidos de letras distintas presentan diferencias significativas, según la prueba de Tukey $(\mathrm{P} \leq 0,05)$, barras verticales indican error estándar $(\mathrm{n}=3)$. 
Los tratamientos con aplicación de termofosfatos presentaron una mayor acumulación de materia fresca en la parte aérea (tallos y foliolos), evidenciando el efecto que tiene la aplicación de P de origen térmico sobre el crecimiento fresco del cultivo. La parte aérea está compuesta por órganos vegetativos, como tallos, hojas y estructuras reproductivas. Las hojas absorben la luz y realizan el intercambio gaseoso, funciones que requieren una presencia activa del fósforo. Según Gómez et al. (2017), la nutrición con P genera un aumento de la masa y volumen de las raíces, dándose un incremento total de la superficie de adsorción de agua y nutrientes, lo que resulta en un incremento del peso fresco de la parte aérea, resultado que se corrobora con lo observado en la figura $2 \mathrm{~A}$.

La acumulación de materia seca por la planta está directamente relacionada con el suministro de nutrientes, siendo la masa seca un indicador de la eficiencia en la absorción de los elementos (Queiroz et al. 2013). La producción de materia seca total es el resultado de la eficiencia del follaje del cultivo en la intercepción y utilización de la radiación solar disponible, durante el ciclo de crecimiento (Santos Castellanos et al. 2010). El mejor indicador de la condición nutrimental de una planta, no es necesariamente el contenido nutrimental del suelo, sino la concentración que demuestra tener la planta después del proceso complicado de la absorción y el transporte interno (Soria, 2008).

El comportamiento de acumulación de materia seca refleja un patrón de tejidos, tal como describió Nkaa et al. (2014). La aplicación de fósforo mejora algunos atributos relacionados con el rendimiento en plantas de Vigna unguiculata, como peso fresco y seco de la vaina, número de vainas, longitud de las vainas, número de semillas, resultados que concuerdan con lo observado en el presente trabajo. Por su parte, Galindo López et al. (2018) observaron que la aplicación de diferentes dosis de un termofosfato generó un aumento del peso fresco y seco, en plantas de fresa.

Contenido de fósforo foliar: Se presentaron diferencias significativas entre tratamientos $(\mathrm{P} \leq 0,05)$. La mayor concentración de $\mathrm{P}$, se obtuvo con la dosis de $300 \mathrm{~kg} \mathrm{ha}^{-1}$, con una media de $0,30 \pm 0,008 \%$, sin diferencias estadísticas con la dosis de $600 \mathrm{~kg} \mathrm{ha}^{-1}$,

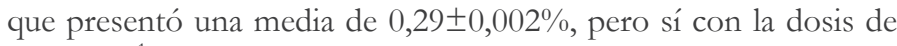
$900 \mathrm{~kg} \mathrm{ha}^{-1}$ y el testigo que presentó la menor concentración, con 0,22 $\pm 0,005 \%$ (Figura 3).

Los resultados indican que el uso del fosfato térmico generó un aumento de la concentración en tejido de fósforo, representado en un incremento en el crecimiento y a productividad de papa, debido a que la forma de fósforo, presente en los fosfatos térmicos, es citrosoluble e hidrosoluble, siendo ideal para condiciones de suelos tropicales, en los cuales, la presencia de acidez intercambiable del suelo, ayuda en el proceso de solubilización del mismo, de una forma gradual, por lo que la disponibilidad de este elemento será durante todo el ciclo fenológico de la planta (Quintana-Blanco et al. 2017).

El fósforo es un elemento esencial para las plantas y se requiere un adecuado suplemento de este nutriente para que la planta crezca y se reproduzca de una manera óptima (Munera Vélez \& Meza Sepúlveda, 2012). Según Marschner (2012), la mayoría de las plantas tiene un adecuado contenido de fósforo cuando, al ser cuantificado en el tejido, se encuentra en un $0,2 \%$ con relación al peso seco; en el presente estudio, el fósforo en tejido foliar presentó una mayor acumulación de fósforo, a nivel foliar. Según Singh et al. (2013), un mayor contenido de fósforo, a nivel de tejido puede tener un efecto directo en la capacidad fotosintética de las plantas. Lo anterior podría explicar el aumento del peso fresco y

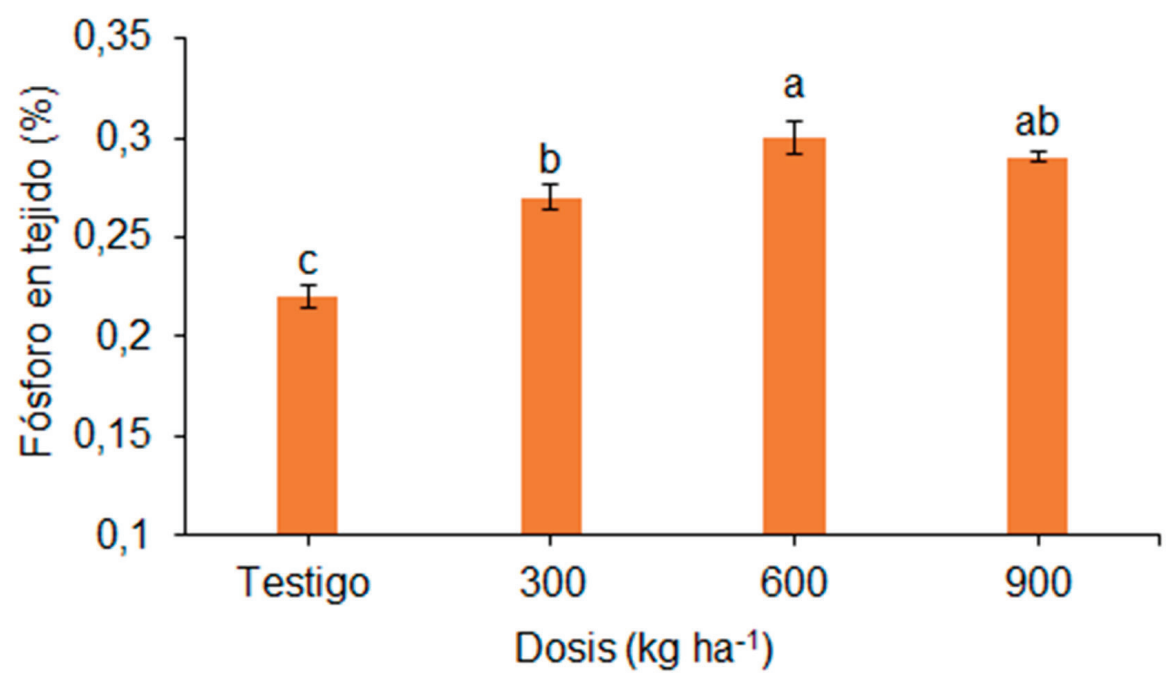

Figura 3. Contenido foliar de fósforo en plantas de papa (Solanum tuberosum L.) con aplicación de termofosfato, en diferentes dosis. Letras distintas entre tratamientos indican diferencias significativas, según la prueba de Tukey $(\mathrm{P}<0,05)$. Barras verticales indican error estándar $(n=3)$. 

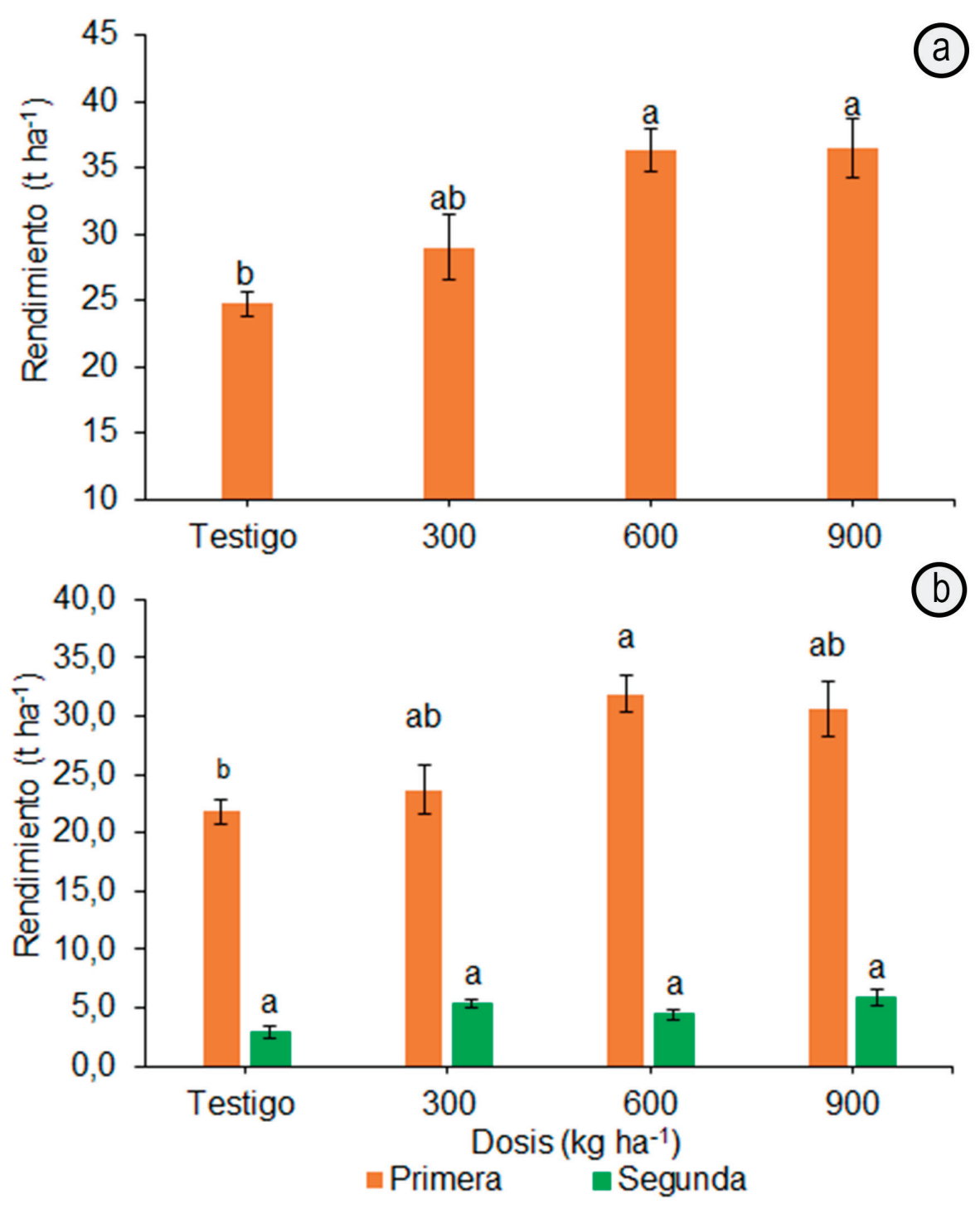

Figura 4. a. Rendimiento total; b. Rendimiento por calidades de papa (Solanum tuberosum L.) con aplicación de termofosfato, en diferentes dosis. Letras distintas entre tratamientos indican diferencias significativas, según la prueba de Tukey $(\mathrm{P}<0,05)$. Barras verticales indican error estándar $(n=3)$.

seco (Figura 1A y B, 2A y B) y el mayor rendimiento (Figura 4), que se observó en este estudio.

Rendimiento: Se presentaron diferencias significativas $(\mathrm{P} \leq 0,05)$, tanto para el rendimiento total como para el rendimiento por calidades. El tratamiento que presentó el mejor rendimiento total fue la dosis de $600 \mathrm{~kg} \mathrm{ha}^{-1}$, con un valor de $36,5 \pm 2,6 \mathrm{t} \mathrm{ha}^{-1}$ frente al testigo, que mostró un rendimiento total de $24,7 \pm 0,8 \mathrm{t} \mathrm{ha}^{-1}$ (Figura 4B). En cuanto al rendimiento por calidad, se presentaron diferencias estadísticas $(\mathrm{P} \leq 0,05)$, para la clasificación primera, que correspondió a tubérculos con diámetros de 65 a $90 \mathrm{~mm}$. El mayor resultado, se obtuvo con el tratamiento correspondiente a la dosis de $300 \mathrm{~kg} \mathrm{ha}^{-1}$, con un valor medio de $31,9 \pm 2,1 \mathrm{t} \mathrm{ha}^{-1}$ frente al testigo, que presentó un valor medio de $21,9 \pm 1,07 \mathrm{tha}^{-1}$. Por su parte, la clasificación segunda, que correspondió a tubérculos con un diámetro de 45 a
$64 \mathrm{~mm}$, no se presentó diferencias estadísticas $(\mathrm{P} \leq 0,05)$; lo anterior indica, una alta relación del contenido de fósforo en tejido y el efecto fisiológico que ejerce en el cultivo (Figura 4A).

El balance nutricional y la oferta de nutrientes en forma oportuna mejoran el área foliar y el rendimiento de tubérculos (Pérez et al. 2008). Según Fernandes et al. (2017), en condiciones de disponibilidad de P baja, media y alta, la fertilización con fosfato logró un aumentó en el crecimiento y diámetro del tubérculo. Este fenómeno también fue descrito en el estudio de encalado y de fertilización fosfatada en el cultivo de papa, en un andosol, de la sierra veracruzana, donde se evidenció un incremento del rendimiento en el cultivo de papa con la aplicación de dosis crecientes de fósforo (Aguilar-Acuña et al. 2003). Según lo mencionado anteriormente y enfatizado por este estudio, la adecuada disponibilidad de fósforo P generó un mejor 
crecimiento y productividad del cultivo de papa Parda Pastusa variedad 'Superior', bajo las condiciones de estudio.

La aplicación de una fuente alterna de fósforo en las concentraciones usadas en esta investigación resultó en un aumento del rendimiento respecto a la aplicación de una fuente convencional. Estos resultados coinciden con lo reportado por Quintana Blanco et al. (2017), quienes reportan un aumento en el rendimiento de frijol cv 'ICA Cerinza', con la aplicación de una dosis de $600 \mathrm{~kg} \mathrm{ha}^{-1}$ de termofosfato. Asimismo, Pinzón et al. (2019), en su estudio sobre Cebolla de Bulbo, reportan que con el uso de termofosfatos, se incrementa el rendimiento y la calidad de la cosecha. Por su parte, Fernández \& Meza (2004) indican que la aplicación roca fosfórica calcinadas, como es el caso del fosfato térmico empleado, generó una mejor disponibilidad de fósforo en el cultivo de maíz, representado en una mayor producción de biomasa en un suelo tropical ácido, que concuerda con los resultados observados en el presente estudio.

Galindo López et al. (2018) encontraron que plantas de fresa aumentaron la producción de frutos y de estructuras reproductivas, al aplicar diferentes dosis de un fosfato térmico, respuesta que asociaron a que, este elemento, es uno de los nutrientes que más requieren las plantas para su desarrollo, formando compuestos relacionados a la base genética de la planta y componentes energéticos del metabolismo vegetal, como ATP y ADP, necesarios como fuente de energía en procesos metabólicos, para la formación de frutos. Romero Romano et al. (2012) evidenciaron que la aplicación de fertilización fosfórica en fresa registró el mejor diámetro ecuatorial y polar, siendo fundamental en la clasificación de fresa por calidades comerciales, resultado que concuerda con los observados en la presente investigación, en la que se observaron tubérculos de mejor diámetro, con la aplicación creciente del fosfato térmico empleado.

El termofosfato que se empleó en el estudio, además de fósforo, aporta nutrientes, como $\mathrm{Ca}, \mathrm{Mg}$, y $\mathrm{Si}$, de gran importancia para la planta; el Ca es un elemento esencial en el desarrollo de diferentes órganos en las plantas, ya que es indispensable para la formación y la multiplicación de las células (Rios Quinchoa et al. 2010). Por su parte, Jerez \& Martín (2012) dicen que el tamaño de los tubérculos depende, principalmente, de factores que afecten la fotosíntesis, como los contenidos de $\mathrm{Mg}$ y $\mathrm{P}$; el $\mathrm{Mg}$ juega un papel importante en la transferencia de fosfatos ricos en energía para el metabolismo del ATP, en donde el P es el elemento constituyente. Esta razón explica el bajo rendimiento en los tratamientos sin aplicación o con aplicación del termofosfato.

La aplicación del termofosfato influyó positivamente en variables de crecimiento y de rendimiento en plantas de papa (S. tuberosum) variedad 'Superior'; los mejores resultados, se obtuvieron con el tratamiento correspondiente a la dosis de $600 \mathrm{~kg} \mathrm{ha}^{-1}$ del termofosfato, convirtiéndolo en una alternativa el suministro de fósforo, en el cultivo de papa variedad Superior, bajo las condiciones de estudio.

De los resultados de esta investigación, se puede concluir que el uso de fosfato térmico puede ser una excelente alternativa al excesivo uso de fertilizantes fosfóricos de alta solubilidad; este último, como fuente finita para la agricultura.

La aplicación de fosfato térmico en el cultivo de papa Parda Pastusa var. Superior puede generar incrementos en rendimiento de hasta 36.5 ton ha ${ }^{-1}$ con respecto al manejo convencional de fertilizantes $\left(24.7\right.$ ton $\left.\mathrm{ha}^{-1}\right)$.

Conflicto de intereses: El manuscrito fue preparado y revisado con la participación de todos los autores, quienes declaramos que no existe ningún conflicto de intereses que ponga en riesgo la validez de los resultados presentados".

\section{REFERENCIAS}

1. AGUILAR-ACUÑA, J.L.; LÓPEZ-MORGADO, R.; NÚÑEZESCOBAR, R.; KHALIL GARDEZI, A. 2003. Encalado y fertilización fosfatada en el cultivo de papa en un Andosol de la Sierra Veracruzana. Terra Latinoamericana. 21(3):417426.

2. ALMEYDA ORTIZ, A.A.; NIÑO DÍAZ, R.A. 2010. Obtención de abono enriquecido a partir de roca fosfórica y Leonardita mediante procesos piro metalúrgicos y químicos. Universidad Industrial de Santander. 66p.

3. CUADROS, G.A.; GÓMEZ S., G.R. 2010. Asociación simbiótica entre hongos micorrízicos arbusculares y el sistema radicular de plántulas de cacao (Theobroma cacao L.): efecto de la formononetina y la disponibilidad de fósforo en el suelo. Corpoica Ciencia Y Tecnología Agropecuaria. 12(1):77-85. https://doi.org/10.21930/rcta.vol12_num1_art:217

4. ESTRADA RAMOS, N. 2000. La biodiversidad en el mejoramiento genético de la papa. Plural Editores. Lima, Perú. ISBN 84-89891-68-0. 372p.

5. FEDERACIÓN COLOMBIANA DE PRODUCTORES DE PAPA, FEDEPAPA. 2019. Departamento de Sistemas de Información y Estudios Económicos - Fondo Nacional de Fomento de la Papa Boletín Mensual Regional Nº 86. Volumen 2.

6. FERNANDES, A.M.; SORATTO, R.P.; SOUZA, E.F.C.; JOB, A.L.G. 2017. Nutrient uptake and removal by potato cultivars as affected by phosphate fertilization of soils with different levels of phosphorus availability. Rev. Bras. Cienc. Solo. 41:e0160288. https://doi.org/10.1590/18069657rbcs20160288

7. FERNÁNDEZ, M. 2007. Fósforo: amigo o enemigo. ICIDCA. Sobre los Derivados de la Caña de Azúcar. 41(2):51-57. 
8. FERNÁNDEZ, S.; NOGUERA, R. 2003. Producción de fosfatos térmicos a partir de rocas fosfóricas nacionales. Agronomía Tropical. 53(1):49-58.

9. FERNÁNDEZ, S.M.; MEZA, C.A. 2004. Efecto residual de la roca fosfórica de riecito modificada por calcinación o acidulación sobre plantas de maíz en suelos con nivel variable de calcio. Bioagro. 16(2):93-98.

10. GAITÁN MORENO, Á.P.; GONZÁLEZ MOGOLLÓN, M.P.A.; NUÚSTEZ LÓPEZ, C.E.; SALDAÑA VILLOTA, T.M.; COTES TORRES, J.M. 2013. Análisis Funcional de Crecimiento y Desarrollo de Cuatro Variedades de Papa (Solanum tuberosum subsp. andigena). Revista Facultad De Ciencias Básicas. 9(2):172-185.

https://doi.org/10.18359/rfcb.344

11. GALINDO LÓPEZ, F.; PINZÓN SANDOVAL, E.; QUINTANA BLANCO, W.; SERRANO, P.; GALÁN, M. 2018. Evaluación de un termofosfato en el crecimiento y producción de fresa (Fragaria x ananassa Duch.) cv. 'Albión'. Rev. U.D.C.A Act. \& Div. Cient. 21(1):61-69. https://doi.org/10.31910/rudca.v21.n1.2018.663

12. GARCÍA, B.; PANTOJA, C. 1998. Fertilización del cultivo de la papa en el departamento de Nariño. p. 8-26. En: Guerrero, R. (ed.). Fertilización de cultivos de clima frío. Segunda edición. Monómeros Colombo-Venezolanos, Bogotá. 370p.

13. GÓMEZ, M.I.; MAGNITSKIY, S.; RODRÍGUEZ, L.E.; DARGHAN, A.E. 2017. Accumulation of N, P, and K in the tubers of potato (Solanum tuberosum L. ssp. andigena) under contrasting soils of the Andean region of Colombia. Agron. Colomb. 35(1):25-35.

https://doi.org/10.15446/agron.colomb.v35n1.61068

14. INTERNATIONAL PLANT NUTRITION INSTITUTE (IPNI). 1999. Better Crops With Plant Food. Phosphorus. 83:1.41p.

15. JEREZ MOMPIES, E.; MARTÍN MARTÍN, R. 2012. Comportamiento del crecimiento y el rendimiento de la variedad de papa (Solanum tuberosum L.) Spunta. Cultivos Tropicales. 33(4):53-58.

16. MARSCHNER, P. 2012. Mineral Nutrition of Higher Plants. 3nd. Edition. Elsevier. Oxford, UK. p.316, 320, 645.

17. MUNERA VÉLEZ, G.A.; MEZA SEPÚLVEDA, D.C. 2012. $\mathrm{El}$ fósforo elemento indispensable para la vida vegetal. Universidad Tecnológica de Pereira. 52p.

18. NKAA, F.; NWOKEOCHA, O.; IHUOMA, O. 2014. Effect of phosphorus fertilizer on growth and yield of cowpea (Vigna unguiculata). J. Pharmacy Biological Sc. 9(5):74-82. https://doi.org/10.9790/3008-09547482
19. ÑÚSTEZ, C.E.; SANTOS, M.; NAVIA, S.L.; COTES, J.M. 2006. Evaluación de la fertilización fosfórica foliar y edáfica sobre el rendimiento de la variedad de papa "Diacol Capiro" (Solanum tuberosum L.). Capiro" (Solanum tuberosum L.). Agron. Colomb. 24(1):16-26.

20. ÑÚSTEZ LÓPEZ, C.E.; SANTOS CASTELLANOS, M.; SEGURA ABRIL, M. 2009. Acumulación y distribución de materia seca de cuatro variedades de papa (Solanum tuberosum L.) en Zipaquirá, Cundinamarca (Colombia). Rev. Facultad Nacional de Agronomía - Medellín. 62(1):48234834.

21. PÉREZ, L.C.; RODRÍGUEZ, L.E.; GÓMEZ, M.I. 2008. Efecto del fraccionamiento de la fertilización con N, P, K y $\mathrm{Mg}$ y la aplicación de los micronutrientes B, Mn y Zn en el rendimiento y calidad de papa criolla (Solanum phureja) variedad Criolla Colombia. Agronomía Colombiana. 26(3):477-486.

22. PINZÓN, E.H.; MUNEVAR, O.E.; TORRES, D.F.; CRUZ, E.F. 2019. Efecto de una fuente alterna de Fósforo en la producción de Cebolla de Bulbo (Allium cepa L.) bajo condiciones de campo. Rev. Investigación Agraria y Ambiental. (Colombia). 10(2):51-61. https://doi.org/10.22490/21456453.2545

23. QUEIROZ, L.J.; ARAUJO Q., A.; BORGES, M.; CAMARGOS O., R.; SOARES L., S.; RIBEIRO C., R. 2013. Influence of phosphate fertilization on phosphorus levels in foliage and tuber yield of the potato cv. Ágata. Semina: Ciências Agrárias. 34(2):649-656. https://doi.org/10.5433/1679-0359.2013v34n2p649

24. QUINTANA BLANCO, W.; PINZÓN SANDOVAL, E.; TORRES, D. 2017. Efecto de un fosfato térmico sobre el crecimiento y producción de fríjol (Phaseolus vulgaris L.) cv Ica Cerinza. Rev. U.D.C.A Act. \& Div. Cient. 20(1):51-59. https://doi.org/10.31910/rudca.v20.n1.2017.62

25. RIOS QUINCHOA, J.Y.; JARAMILLO VILLEEGAS, S. DEL C.; GONZÁLEZ SANTAMARÍA, L.H.; COTES TORRES, J.M. 2010. Determinación del Efecto de Diferentes Niveles de Fertilización en Papa (Solanum tuberosum ssp. andigena) DIACOL Capiro en un suelo con propiedades ándicas de Santa Rosa de Osos, Colombia. Rev. Fac. Nal. Agr. Medellín. 63(1):5225-5237.

26. ROBERTS, T.L.; JOHNSTON, A.E. 2015. Phosphorus use efficiency and management in agriculture. Resources, Conservation and Recycling, 105:275-281. https://doi.org/10.1016/j.resconrec.2015.09.013

27. RODRÍGUEZ, J.A.; RODRÍGUEZ, E.M.M. 2014. Producción integrada de papa (Solanum tuberosum L.): un análisis de 
sensibilidad agroeconómica para el sudeste bonaerense, Argentina. Rev. Latinoam. de la Papa. 18(2):20-35.

28. ROMERO-ROMANO, C.O.; OCAMPO-MENDOZA, J.; SANDOVAL-CASTRO, E.; TOBAR-REYES, J.R. 2012. Fertilización orgánica-mineral y orgánica en el cultivo de fresa (Fragaria $\mathrm{x}$ ananassa Duch.) bajo condiciones de invernadero. Rev. Ra Ximhai. 8(3):41-49.

https://doi.org/10.35197/rx.08.03.e1.2012.04.cr

29. SANTOS CATELLANOS, M.; SEGURA ABRIL, M.; NUÚSTEZ LÓPEZ, C.E. 2010. Análisis de crecimiento y relación fuente-demanda de cuatro variedades de papa (Solanum tuberosum L.) en el municipio de Zipaquirá (Cundinamarca, Colombia). Rev. Fac. Nal. Agr. Medellín. 63(1):5253-5266.
30. SINGH, S.K; BADGUJAR, G.B.; REDDY, V.R.; FLEISHER, D.H.; TIMLIN, D.J. 2013. Effect of phosphorus nutrition on growth and physiology of cotton under ambient and elevated carbon dioxide. J. Agron. Crop Sci. 199:436-448. https://doi.org/10.1111/jac.12033

31. SORIA I., N. 2008. Nutrición Vegetal y Defensa Natural. En: Memorias: Sociedad Ecuatoriana de la Ciencia del suelo, XV Congreso de la Ciencia Ecuatoriana del Suelo, Quito 2008. p.1-11.

32. TAIZ, L.; ZEIGER, E. 2010. Plant Physiology. $5^{\text {th }}$ ed. Sinauer Associates Inc., Publishers Sunderland, Massachusetts U.S.A. 778p. 